\title{
Der Einfluß der Umgebungstemperatur auf Motilität, Körpertemperatur und Sauerstoffverbrauch von normalen und Pervitin-behandelten Mäusen ${ }^{1}$
}

\author{
FrIEDRICH K. OHNESORGE \\ Pharmakologisches Institut der Universität Kiel
}

\begin{abstract}
The effect of the ambient temperature on motility, body temperature and oxygen consumption of normal and methamphetamine-treated mice. Single mice were kept in various ambient temperatures $\left(15^{\circ}\right.$ to $\left.35^{\circ} \mathrm{C}\right)$ and motility, oxygen consumption, and body temperature were recorded. Untreated animals: Motility was least at $25^{\circ} \mathrm{C}$ room temperature. Relations between motility and body temperature were linear at all ambient temperatures. The body temperatures of very agile mice did not vary at ambient temperatures from $15^{0}$ to $30^{\circ} \mathrm{C}$ whereas that of quiet mice was strongly influenced by the milieu. The relations between oxygen consumption and body weight were also linear at all ambient temperatures; the corresponding regression coefficients decreased progressively with rising ambient temperatures. Oxygen consumption increased at a constant rate with motility, independent of ambient temperatures. Animals treated with methamphetamine: The $\mathrm{LD}_{\overline{0} 0}$ of methamphetamine decreased considerably with rising ambient temperature. The influence on body temperature of methamphetamine was very variable and depended on both dose and ambient temperature. Toxic doses of methamphetamine induced hyperthermia in warm surroundings and hypothermia in a cool milieu. Under the influence of methamphetamine, oxygen consumption increased or decreased considerably with the body temperature. Ambient temperatures exerted an essential influence on the cause of death after toxic doses of methamphetamine.
\end{abstract}

\section{EINLEITUNG}

Usinger (1957) untersuchte das Verhalten von Körpertemperatur und den $\mathrm{O}_{g^{-}}$ Verbrauch an Mäusen, die an die jeweilige Umgebungstemperatur (UT) adaptiert waren. Über die akuten Veränderungen von Motilität, Körpertemperatur und $\mathrm{O}_{2}$-Verbrauch bei Mäusen, die einzeln verschiedenen UT ausgesetzt worden sind, ist bisher nichts bekannt.

Wir benötigten diese Werte, um den Einfluß der UT auf die entsprechenden Wirkungen von Pervitin (Methamphetamin) beurteilen zu können. Es ist bekannt, daß die Toxität und die zentralerregenden Wirkungen dieses Weckamins durch zahlreiche äußere Faktoren verändert werden können (CHANCE 1946, 1947; LASAGNA \& MCCANN 1957; Höhn \& Lasagna 1960; Askew 1961, 1962; Wetss et al. 1961; Greenblatt \&

1 Die ausfuhrliche Darstellung der Ergebnisse erfolgt an anderer Stelle. 
Osterberg 1961; Fink \& Larson 1962; Weaver \& Kerley 1962), man weiß bisher aber noch nicht, wie die UT die Toxicität von Pervitin und seine Wirkungen auf den Gasstoffwechsel und die Körpertemperatur bei einzeln sitzenden Mäusen verändert.

\section{METHODIK}

Verwendet wurden männliche NMRI-Mäuse, die in Sammelkäfigen bei $21^{\circ}-25^{\circ} \mathrm{C}$ aufbewahrt wurden. Während der Versuche wurden Futter und Wasser entzogen. Die Versuche wurden in UT von $15^{\circ}, 20^{\circ}, 25^{\circ}, 30^{\circ}$ und $35^{\circ}\left( \pm 0,1^{\circ}\right)$ durchgeführt.

Erste Versuchsreihe: Die Mäuse wurden einzeln in Glaszylinder gesetzt. Thermoelektrische Messung der Rektaltemperatur am Anfang und 1, 21/2, 31/2 und 5 Stunden nach Versuchsbeginn. Unmittelbar vor jeder Temperaturmessung wurde im Abstand von $1 \mathrm{~min} 10 \mathrm{mal}$ entschieden, ob sich eine Maus bewegte oder nicht bewegte. Wir erhielten dadurch eine Skala für die Bewegungshäufigkeit von 1-10. Nach 21/2 Stunden wurde eine $0,9 \%$ ige $\mathrm{NaCl}$ - oder Pervitin-Lösung subcutan injiziert.

Zweite Versuchsreihe: Fortlaufende Registrierung des $\mathrm{O}_{2}$-Verbrauchs in 10-minPerioden. Gleichzeitige Messung der Laufaktivität mit einem Laufrad. Nach 21/2 Stunden subcutane Injektion von $\mathrm{NaCl}$ oder Pervitin, Abbruch des Versuchs nach 5 Stunden.

\section{ERGEBNISSE}

\section{Unbehandelte Mäuse}

Die mittlere Körpertemperatur (KT) bei Versuchsbeginn beträgt $36,43 \pm 0,05^{\circ} \mathrm{C}$. Nach einstündiger Isolierung steigt die KT in allen Umgebungstemperaturen (UT) um $1-2^{0}$ an; die mittlere Bewegungshäufigkeit ist hoch (7-9) und zwischen den einzelnen UT-Gruppen unregelmäßig verteilt.

Nach 21/2 Stunden hat sich die mittlere KT der Gruppen (je 230 Tiere) in 15-250 UT auf $36,6^{\circ}$ eingestellt (in $15^{\circ} U T 36,62 \pm 0,07^{\circ}$, in $20^{\circ} U T 36,58 \pm 0,05^{\circ}$, in $25^{\circ} U T$ $36,60 \pm 0,06^{\circ}$ ). In $30^{\circ} \mathrm{UT}$ ist die KT mit $36,82 \pm 0,06^{\circ}$ leicht und in $35^{\circ}$ mit $38,35 \pm$

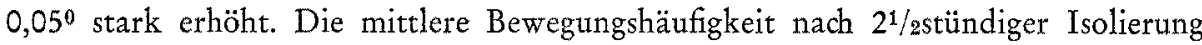
ist in einer U'T von $25^{\circ}$ am geringsten, sie steigt in kühlerer und wärmerer Umgebung zunehmend an (mittlere Bewegungshäufigkeit in $15^{\circ}$ UT 5,51, in $20^{\circ}$ UT 4,90, in $25^{\circ} U T 2,50$, in $30^{\circ} U T 3,05$, in $35^{\circ}$ UT 5,61).

Dem Minimum der mittleren Bewegungshäufigkeit in $25^{\circ}$ U'T entspricht, daß auch die prozentuale Häufigkeit der Mäuse mit sehr niedriger Bewegungshäufigkeit (0-1) in $25^{\circ}$ UT am größten ist, und in wärmerer und kälterer Umgebung abnimmt (relativer Anteil der Mäuse mit Bewegungshäufigkeit 0-1: in 150 UT $27,7 \%$, in $20^{\circ}$ UT 31,9\%, in 250 UT 66,3\%, in $30^{\circ}$ UT 52,8\%, in 350 UT 23,7\%). Der Prozentsatz der Mäuse mit hoher Bewegungshäufigkeit verhält sich spiegelbildlich (Bewegungshäufigkeit 8-10: in $15^{\circ}$ UT $42,8 \%$, in $200^{\circ}$ UT $36,6 \%$, in $25^{\circ}$ UT $15,6 \%$, in $30^{\circ}$ UT $20,0 \%$, in $35^{\circ}$ UT $36,4 \%$. Signifikante Unterschiede in der motorischen Aktivität bestehen zwischen 35 und $30^{\circ}$ UT und 25 und $20^{\circ}$ UT. 
Zwischen Bewegungshäufigkeit und KT der Mäuse bestehen in allen UT eindeutige lineare Beziehungen. Die Regressionsgeraden verlaufen zwischen 20 und $30^{\circ}$ UT zunehmend flacher; bei Zunahme der Bewegungshäufigkeit um den Wert 1 steigt also die KT in $20^{\circ}$ UT stärker an als in $30^{\circ}$ UT. Die Gerade für $15^{\circ}$ UT liegt parallel unter der für $20^{\circ}$ und die Gerade für $35^{\circ}$ UT parallel über der für $30^{\circ}$. In UT von 15-300 besitzen sehr agile Mäuse annähernd die gleiche KT (errechnete Werte für Bewegungshäufigkeit 10: in $15^{\circ}$ UT $37,52^{\circ}$, in $20^{\circ}$ UT $37,55^{\circ}$, in $25^{\circ}$ UT $37,66^{\circ}$, in $30^{\circ}$ UT $37,62^{\circ}$, in $35^{\circ}$ UT $38,82^{\circ}$ ), während ruhige Mäuse deutlich abweichende KT haben (er-

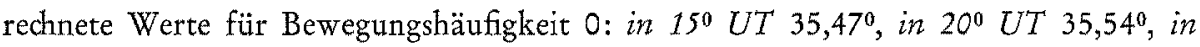
$25^{\circ} U T 36,22^{\circ}$, in $30^{\circ} U T 36,45^{\circ}$, in $35^{\circ}$ UT 37,600). Die Regressionskoeffizienten in den verschiedenen UT haben folgende Größen ( $\mathrm{r}^{2}=$ Bestimmtheitsmaß): in $15^{\circ} \mathrm{UT}$ $0,205^{0} /$ Mot $\left(\mathrm{r}^{2}=0,972\right)$, in $20^{\circ}$ UT 0,2010/Mot $\left(\mathrm{r}^{2}=0,932\right)$, in $25^{\circ}$ UT 0,1440/Mot $\left(\mathrm{r}^{2}=0,977\right)$, in $30^{\circ} U T$ T,1170/Mot $\left(\mathrm{r}^{2}=0,990\right)$, in $35^{\circ}$ UT 0,1220/Mot $\left(\mathrm{r}^{2}=0,974\right)$.

Wir müssen diesen Befunden wohl entnehmen, daß die Mäuse ihre KT mit Hilfe der Motilität auf den ursprünglichen Soll-Wert, der bei $36,6^{\circ}$ liegt, einstellen können. Diese Regelung versagt bei UT von $30^{\circ}$ und darüber, weil der durch die Wärme ausgelöste Fluchttrieb offenbar stärker ist als die Fähigkeit, die Motorik zur Regulierung der KT zu dämpfen. - Wenn wir zur Erklärung der verschiedenen Neigungen der Regressionsgeraden annehmen, daß pro Punkt Bewegungshäufigkeit in allen UT die gleiche Wärmemenge mehr produziert wird, dann muß die Wärmeabgabe mit zunehmender Bewegungshäufigkeit in der Kälte weniger ansteigen als in der Wärme. Die Parallelverschiebung der Geraden in 15 und $35^{\circ}$ UT zeigt, daß die Drosselung der regulierbaren Wärmeabgabe bereits in einer UT von $20^{\circ}$ maximal ist; in einer UT von $30^{\circ}$ sind dagegen die Wärmeabgabe-fördernden Mechanismen bereits vollständig in Anspruch genommen.

Zwischen $\mathrm{O}_{2}$-Verbrauch und Körpergewicht bestehen in allen UT lineare Beziehungen. Die Regressionsgeraden verlaufen mit steigender UT (15-30 ) zunehmend flacher; der $\mathrm{O}_{2-}$ Verbrauch nimmt also mit wachsendem Körpergewicht in niedrigen UT stärker zu als in der Wärme. Ist die UT höher als $30^{\circ}$, dann gewinnt das Körpergewicht wieder einen stärkeren Einfluß auf den $\mathrm{O}_{2}$-Verbrauch, wahrscheinlich bedingt durch die starke motorische Unruhe in warmer Umgebung. Die Gleichungen der Regressionsgeraden $(y=a+b x)$ in den verschiedenen UT lauten $\left(r^{2}=\right.$ Bestimmtheitsmaß): in $15^{\circ}$ UT y $=7,78+0,476 \mathrm{x}\left(\mathrm{r}^{2}=0,841\right)$, in $20^{\circ}$ UT $\mathrm{y}=7,69+0,363 \mathrm{x}\left(\mathrm{r}^{2}=0,803\right)$, in $25^{0} U T \mathrm{y}=9,85+0,153 \mathrm{x}\left(\mathrm{r}^{2}=0,984\right)$, in $30^{0} U T \mathrm{y}=8,81+0,084 \mathrm{x}\left(\mathrm{r}^{2}=\right.$ $0,848)$, in $35^{\circ} U T \mathrm{y}=2,45+0,334 \mathrm{x}\left(\mathrm{r}^{2}=0,980\right)$, wobei der Regressionskoeffizient $\mathrm{b}$ die Dimension $\mathrm{ml} \mathrm{O}_{2} / 10$ min $\times \mathrm{g}$ Körpergewicht hat und $\mathrm{y}$ den $\mathrm{O}_{2}-\mathrm{Verbrauch} / 10 \mathrm{~min}$ beim Körpergewicht $\mathrm{x}$ angibt. Die den Gleichungen zugrunde liegenden Werte wurden ermittelt aus dem individuell kleinsten, während einer 10 min-Periode beobachteten $\mathrm{O}_{2}$-Verbrauch während der ersten $21 / 2 \mathrm{Std}$. des Versuches. Pro UT wurden 50-75 Tiere mit Gewichten zwischen 20 und $38 \mathrm{~g}$ eingesetzt.

Pro g Körpergewicht berechnet verbrauchen schwere Mäuse weniger $\mathrm{O}_{2}$ als leichte Mäuse. Die absolute Abnahme des $\mathrm{O}_{2}$-Verbrauchs ist in UT von 15-30 annähernd gleich groß, in $35^{\circ}$ jedoch wesentlich kleiner (minimaler $O_{g}$-Verbrauch pro g Maus und $10 \mathrm{~min}$ für $20 \mathrm{~g}$ und $38 \mathrm{~g}$ schwere Tiere: in $15^{\circ}$ UT 0,830 und 0,667 , in $20^{\circ}$ UT 
0,718 und 0,553 , in $25^{\circ}$ UT 0,605 und 0,391 , in $30^{\circ}$ UT 0,494 und 0,296 , in $35^{\circ}$ UT $0,446$ und 0,349$)$.

Der $\mathrm{O}_{2}$-Verbrauch wächst mit steigender Laufleistung im Laufrad in allen UT annähernd um den gleichen Betrag. Der mittlere $\mathrm{O}_{2}$-Verbrauch/30 min von Mäusen, die während dieser Zeit nicht (Gruppe I), 2,35-18,80 m (Gruppe II) und mehr als $21,5 \mathrm{~m}$ (Gruppe III) gelaufen sind, beträgr: in $15^{\circ}$ UT Gr. I 71,18 ml, Gr. II 75,35 ml, Gr. III $87,00 \mathrm{ml}$; in $20^{\circ}$ UT Gr. I $59,44 \mathrm{ml}$, Gr. II $63,93 \mathrm{ml}$, Gr. III 72,11 ml; in $25^{\circ}$ UT Gr. I 42,99 ml, Gr. II 48,24 ml, Gr. III 60,51 ml; in $30^{\circ}$ UT Gr. I $36,20 \mathrm{ml}$, Gr. II 41,86 ml, Gr. III 53,77 ml. Durch die weitere Aufschlüsselung dieser Werte läßt sich zeigen, daß der Anteil der nicht registrierten Bewegungen wie Aufrichten, Putzen, Zittern usw. am gesamten $\mathrm{O}_{2}$-Verbrauch in UT von 20,25 und $30^{\circ}$ bei leichten und schweren Mäusen etwa gleich, in $15^{\circ} \mathrm{UT}$ aber bei leichten Tieren wesentlich größer ist als bei schweren Tieren.

\section{Versuche mit Pervitin}

Pervitin wirkt um so giftiger, je höher die UT ist. Die mittlere letale Dosis $\left(\mathrm{LD}_{50}\right)$ beträgt in $15^{\circ}$ UT 203,0 (191,1-215,6) $\mathrm{mg} / \mathrm{kg}$; in $20^{\circ}$ UT 180,0 $(167,4-193,5) \mathrm{mg} / \mathrm{kg}$; in $25^{\circ}$ UT 142,0 (133,6-151,0) mg/kg; in $30^{\circ}$ UT 42,2 (32,2-55,3) $\mathrm{mg} / \mathrm{kg}$; in $35^{\circ}$ UT $9,5(8,2-11,0) \mathrm{mg} / \mathrm{kg}$ bei subcutaner Injektion und $2^{1} / 2$ stündiger Beobachtungsdauer. Die mittleren letalen Dosen und die Neigungen der Dosiswirkungsgeraden für benachbarte UT unterscheiden sich voneinander signifikant $(p<0,05)$. Die Todesursache ist demnach für jede UT eine andere.

Die KT wird je nach Dosis und UT durch Pervitin unterschiedlich beeinflußt. 1 Std. nach der Injektion verursachen $3 \mathrm{mg} / \mathrm{kg}$ in 35 und $30^{\circ} \mathrm{UT}$ keine Anderung der $\mathrm{KT}$, in $15-25^{\circ}$ UT eine signifikante Senkung der KT (in $35^{\circ}$ UT Kontr. 38,240, Pervitin $38,40^{\circ}$; in $30^{\circ}$ UT Kontr. 36,96\%, Pervitin 36,800; in $25^{\circ}$ UT Kontr. 36,740, Pervitin $35,66^{\circ}$; in $20^{\circ}$ UT Kontr. $35,92^{\circ}$, Pervitin $34,41^{\circ}$; in $15^{\circ}$ UT Kontr. $37,17^{0}$, Pervitin $\left.34,93^{\circ}\right)$. Höhere Dosen $\left(5-20 \mathrm{mg} / \mathrm{kg}\right.$ ) rufen in UT von $25-35^{\circ}$ Hyperthermie hervor, die um so stärker ist, je höher Dosis und UT sind; sie bewirken in UT von 15 und $20^{\circ}$ einen Wiederanstieg der KT. Nach toxischen Dosen (siehe oben) sterben die Mäuse in 35 und $30^{\circ}$ UT im hyperthermen Zustand, in $25^{\circ} \mathrm{UT}$ bei etwa normaler KT und in

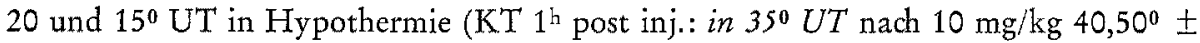
$0,22^{\circ}$; in $30^{\circ}$ UT nach $40 \mathrm{mg} / \mathrm{kg} 38,79^{\circ} \pm 0,21^{\circ}$; in $25^{\circ}$ UT nach $150 \mathrm{mg} / \mathrm{kg} 36,85^{\circ} \pm$ $0,20^{\circ}$; in $20^{\circ} \mathrm{UT}$ nach $170 \mathrm{mg} / \mathrm{kg} 33,57^{\circ} \pm 0,65^{\circ}$; in $15^{\circ} U T$ nach $200 \mathrm{mg} / \mathrm{kg} 28,85^{\circ}$ $\left.\pm 0,87^{0}\right)$.

$2^{1 / 2}$ Stunden nach der Injektion klingen die Wirkungen von Dosen bis $100 \mathrm{mg} / \mathrm{kg}$ in allen UT wieder $a b$, höhere Dosen (in UT von 20 und $15^{\circ}$ ) haben eine noch stärkere hypotherme Wirkung als $1 \mathrm{Std}$. nach der Injektion.

Der Verlauf der Dosiswirkungskurven für die KT zeigt, daß es sich nicht um eine einfache, mit steigender Dosis zunehmende Lähmung der Thermoregulation handeln kann. Ein Vergleich $z$ wischen den nach 1 und $2^{1 / 2}$ Std. gemessenen Werten schließt aus, daß unterschiedliche Resorptionsgeschwindigkeiten beim Zustandekommen dieser Werte eine wesentliche Rolle gespielt haben. Bei den rach $2 \frac{1}{2}$ Std. gemessenen KT müssen 
die je nach $\mathrm{KT}$ verschiedenen Exkretionsgeschwindigkeiten berücksichtigt werden (verzögerte Exkretion bei hypothermen Tieren, siehe FuHrman und FunRman 1961) und die Tatsache, daß zu diesem Zeitpunkt die Mäuse mit den höchsten KT in warmer Umgebung bereits gestorben sind.

Zwischen den Wirkungen von Pervitin auf die KT und den Wirkungen auf den $\mathrm{O}_{2}$-Verbrauch besteht eine befriedigende Parallelität. In UT von 15 und $20^{\circ}$ verursachen kleine Dosen (1-3 $\mathrm{mg} / \mathrm{kg}$ ) eine teils unsichere, teils signifikante Senkung des $\mathrm{O}_{2}$-Verbrauches; höhere Dosen führen nach vorübergehendem Wiederanstieg schließlich zu einer weiteren Herabsetzung. In wärmerer Umgebung (25 und $30^{\circ}$ ) führt Pervitin lediglich zu einer Steigerung des $\mathrm{O}_{9}-$ Verbrauchs; diese Steigerung setzt mit wachsender UT bei zunehmend kleineren Dosen ein und erreicht absolut höhere Werte $\left(\mathrm{O}_{2}\right.$-Verbrauch zwischen der 40. und 130. min post inj., bezogen auf maximalen $\mathrm{O}_{2-}$ Verbrauch in der Vorperiode: in 150 UT Kontr. $79,9 \% \pm 2,2 \%$, nach $3 \mathrm{mg} / \mathrm{kg}$ $73,20 \% \pm 6,2 \%$, nach $20 \mathrm{mg} / \mathrm{kg} \mathrm{70,7 \%} \pm 5,5 \%$, nach $80 \mathrm{mg} / \mathrm{kg} \mathrm{61,6 \%} \pm 5,7 \%$; in $20^{\circ} U T$ Kontr. $79,6 \% \pm 1,4 \%$, nach $3 \mathrm{mg} / \mathrm{kg} 68,0 \% \pm 4,2 \%$, nach $20 \mathrm{mg} / \mathrm{kg}$ $83,7 \% \pm 2,6 \%$, nach $80 \mathrm{mg} / \mathrm{kg} 102,1 \% \pm 7,8 \%$; in 250 UT Kontr. $71,3 \% \pm 2,6 \%$, nach $3 \mathrm{mg} / \mathrm{kg} 79,9 \% \pm 4,4 \%$, nach $20 \mathrm{mg} / \mathrm{kg} 123,2 \% \pm 6,3 \%$, nach $80 \mathrm{mg} / \mathrm{kg}$ $129,0 \% \pm 7,4 \%$; in $30^{\circ}$ UT Kontr. $60,9 \% \pm 2,4 \%$, nach $3 \mathrm{mg} / \mathrm{kg} 77,2 \% \pm 4,6 \%$, nach $20 \mathrm{mg} / \mathrm{kg} 145,2 \% \pm 6,9 \%$ ).

Die am $\mathrm{O}_{2}$-Verbrauch gemessenen Veränderungen der Wärmebildung sind wahrscheinlich die Ursache und nicht die Folge der Pervitinwirkung auf die KT. Der je nach UT unterschiedliche und außerdem noch irreguläre Verlauf der Dosiswirkungskurve legt die Vermutung nahe, daß es mindestens 2 verschiedene zentrale Angriffspunkte gibt, deren Ansprechbarkeit für Pervitin von der UT abhängt. Möglicherweise sprechen das Kühl- und das Wärmezentrum je nach ihrer Aktivierung durch die jeweils herrschende UT verschieden stark auf Pervitin an. Inwieweit durch Pervitin induzierte Änderungen der motorischen Aktivität den $\mathrm{O}_{2}$-Verbrauch und die $\mathrm{KT}$ beeinflußt haben, bleibt ungewiß. Im Gegensatz zu früheren Untersuchungen (OHNESORGE \& KHAN 1961) lieferte die Laufradmethode in den hier besprochenen Versuchen keine genügend genauen Ergebnisse, um die Zusammenhänge zwischen motorischer Aktivität und $\mathrm{O}_{2}-$ Verbrauch nach Pervitin beurteilen zu können.

\section{ZUSAMMENFASSUNG}

Es wurde untersucht, wie sich Motilität, $\mathrm{O}_{2}$-Verbrauch und Körpertemperatur von normalen und Pervitin- (Methamphetamin-)behandelten Mäusen verändern, wenn diese einzeln verschiedenen Umgebungstemperaturen $\left(15^{\circ}\right.$ bis $35^{\circ} \mathrm{C}$ ) ausgesetzt werden. A. Unbehandelte Mäuse:

1. Die mittlere Bewegungshäufigkeit ist in $25^{\circ} \mathrm{C}$ am kleinsten.

2. Zwischen Bewegungshäufigkeit und Körpertemperatur bestehen in allen Umgebungstemperaturen lineare Beziehungen. In Umgebungstemperaturen von 15 bis $30^{\circ} \mathrm{C}$ haben sehr unruhige Mäuse die gleiche mittlere Körpertemperatur, während bei ruhigen Mäusen die Körpertemperaturen verschieden sind.

3. $\mathrm{O}_{2}$-Verbrauch und Körpergewicht sind in allen Umgebungstemperaturen durch 
einfache lineare Korrelationen miteinander verbunden. Die korrespondierenden Regressionsgeraden verlaufen mit steigender Umgebungstemperatur zunehmend flacher.

4. Der $\mathrm{O}_{2}$-Verbrauch wächst mit steigender Laufleistung in allen Umgebungstemperaturen um annähernd den gleichen Betrag.

B. Versuche mit Pervitin:

1. Die $L D_{50}$ von Pervitin bei subcutaner Injektion sinkt mit wachsender Umgebungstemperatur stark ab.

2. Die Körpertemperatur wird je nach Dosis und Umgebungstemperatur unterschiedlich beeinflußt.

3. Toxische Dosen verursachen in warmer Umgebung Hyperthermie, in kühler Umgebung Hypothermie.

4. Der $\mathrm{O}_{2}$-Verbrauch wird durch Pervitin im gleichen Sinne wie die Körpertemperatur beeinflußt, er steigt mit wachsender und fällt mit sinkender Körpertemperatur.

5. Die Todesursachen sind je nach Umgebungstemperatur verschieden.

\section{ZITIERTE LITERATUR}

Askew, B. M., 1961. Amphetamine toxicity in aggregated mice. J. Pharm. Pharmacol. 13, 701-703.

- 1962. Hyperpyrexia as an contributory factor in the toxicity of amphetamine to aggregated mice. Brit. J. Pharmacol. 19, 245-257.

Chance, M. R. A., 1946. Aggregation as a factor influencing the toxicity of sympathomimetic amines in mice. J. Pharmacol. 87, 214-219.

- 1947. Factors influencing the toxicity of sympathomimetic amines to solitary mice. J. Pharmacol. 89, 289-296.

FINK, G. B. \& LARSON, R. E., 1962. Some determinants of amphetamine toxicity in aggregated mice. J. Pharmacol, 137, 361-364.

Greenblatt, E. N. \& Osterberg, A. C., 1961. Correlation of activating and lethal effects of excitatory drugs in grouped and isolated mice. J. Pharmacol. 131, 115-119.

HöHN, R. \& LASAGNA, L., 1960. Effects of aggregation and temperature on amphetamine toxicity in mice. Psychopharmacologia 1, 210-220.

LASAGNA, L. \& MCCANN, W. P., 1957. Effect of "transquillizing" drugs on amphetamine toxicity in aggregated mice. Science 125, 1241-1243.

OhNesorge, F. K. \& Kran, A. L., 1961. Zur motilitäts- und stoffwechselsteigernden Wirkung von Phenmetrazin. Arzneimittel-Forsch. 11, 793-796.

UsINGER, W., 1957. Respiratorischer Stoffwechsel und Körpertemperatur der weißen Maus in thermoindifferenter Ungebung. Pflügers Arch. ges. Physiol. 264, 520-535.

WeAver, L. C. \& KerLeY, T. L., 1962. Strain differences of mice to d-amphetamine. J. Pharmacol. 135, 240-244.

Werss, B., Latres, V. G. \& Blanton, F. L., 1961. Amphetamine toxicity in rats and mice subjected to stress. J. Pharmacol. 132, 366-371. 


\section{Diskussion im Anschluß an den Vortrag OHNEsorge}

HeusNer: Wenn Sie die Akrivität in Abbängigkeit vom Sauerstoffverbrauch auftragen, können Sie an Hand dieser Funktion einen Ruheenergiewechsel finden, der mit dem direkt gemessenen übereinstimmt?

OHNESORge: Das ist schwer zu sagen. Ich glaube kaum; Untersuchungen darüber habe ich nicht durchgeführt. Wenn eine Maus nicht läuft, kann man nicht sagen, daß sie ohne Aktivität ist, etwa ohne Muskelspannung. Frühere Untersuchungen haben ergeben, daß das Laufen im Laufrad nur $1 / 10$ der Energie benötigt wie das Laufen auf der Ebene. Das entspricht also einem Fahrradeffekt (OHNESORge $\&$ Kran).

Heusner: Wie verläuft dann die Kurve?

OHNESORGE: Die Kurve verläuft linear.

HeUsNer: In welcher Größenordnung liegt der Grundumsatz?

Ohnesorge: Das weiß ich nicht. Die von mir angegebenen Werte für den minimalen $\mathrm{O}_{2}$-Verbrauch (Seite 300) wurden nicht unter den klassischen Bedingungen (Nahrungskarenz usw.) gewonnen.

VON BERTALANFFY: It has perhaps been noticed that we have performed somewhat similar experiments as has Dr. OHNEsorge. We too studied the size relation of metabolic rate under conditions of basal metabolism and muscular activity (Fig. 7) and found that the slope of the regression line with regard to weight decreases with absolute increase of metabolic rate.

OHNESORge: Ja, mit Zunahme der Aktivität wird die Kurve immer steiler.

LOCKER: Ich möchte in diesem Zusammenhang darauf hinweisen, daß Herr OHNESORge ja seine Kurven arithmetisch aufgezeichnet hat, während die von Hernn vON BERTALANFFY logarithmisch aufgetragen wurden. Die beiden Darstellungen lassen sich also nicht ohne weiteres vergleichen. Wäre es nicht möglich, daß Sie, Herr OHNEsorge, da Sie ja ein umfangreiches Material verarbeitet haben, Ihre Ergebnisse in logarithmische Werte umrechnen könnten?

OHNEsoRge: Das geht natürlich. Idh werde Ihnen gern mein Material zur Verfügung stellen. Ich bin an diesen Problemen vor allem als Pharmakologe interessiert gewesen. 